\title{
ELECTRON SCATTERING CROSS-SECTIONS FOR PARTICLE TRANSPORT MODELING IN A WEAKLY IONIZED AIR PLASMA
}

\author{
V.S. ZAKHAROV ${ }^{1}$, M.E. ZHUKOVSKIY ${ }^{1 *}$, S.V. ZAKHAROV ${ }^{2,3}$, M.B. MARKOV ${ }^{1}$ \\ ${ }^{1}$ Keldysh Institute of Applied Mathematics RAS, Miusskaya sq. 4, Moscow, Russia \\ ${ }^{2}$ NRC "Kurchatov Institute", Akademika Kurchatova sq. 1, Moscow, Russia \\ ${ }^{3}$ EATS, Orsay, France \\ *Corresponding author. E-mail: usermath@ mail.ru
}

\section{DOI: 10.20948/mathmontis-2021-51-7}

\begin{abstract}
Summary. Data on processes of electron scattering on ions and neutral atoms are required in fundamental studies and in applied research in such fields as astro- and laser physics, low density plasma simulations, kinetic modeling etc. Experimental and computational data on elastic and inelastic electron scattering in a wide range of electron energies is available mostly for the electron interaction with neutral atoms, but are very limited for the scattering on ions, notably for elastic processes. In present work the calculational approaches for the crosssection computation of electron elastic and inelastic scattering on neutral atoms and ions are considered. The atomic and ion properties obtained in quantum-statistical Hartree-Fock-Slater model are used in the direct computation of electron elastic scattering and ionization crosssections by a partial waves method, semiclassical and distorted-wave approximations. Calculated cross-sections for elastic scattering on nitrogen and oxygen atoms and ions, and electron ionisation cross-sections are compared with the available experimental data and widely used approximations and propose consistent results. Considering applicability of Hartree-Fock-Slater model in wide scope of temperatures and densities, such approach to the cross-section calculation can be used in a broad range of energies and ion charges.
\end{abstract}

\section{INTRODUCTION}

Data on the cross sections of electron scattering by atoms and ions are of considerable interest, both in the field of fundamental science for an in-depth study of interaction processes, and in the applications, and are necessary in many areas of research, such as modeling low-temperature plasma, astrophysics, laser physics, atmospheric effects etc. An example is the interaction of electromagnetic radiation with gas-plasma formations. Plasma layers arise, for example, around of space capsule due to the heating and following ionization of the air [1]. The presence of the plasma affects the propagation of electromagnetic waves. The electromagnetic field impacts the energy of free electrons in the plasma and can change its parameters. The studies of such processes by means of mathematical modeling within the framework of kinetic models require consideration of collisions of electrons not only with neutral molecules of air gases, but also with the nitrogen and oxygen ions etc.

Further to natural phenomena, the processes of collisions of electrons with matter act an essential or major role in plasma technologies, such as, for example, microelectronics [2], radiation sources [3] and biomedicine [4]. Atomic physics, plasma physics and optics are the domains directly related to electron-atomic and electron-ion collisions contribute significantly

2010 Mathematics Subject Classification: 82D10, 81T80, 97M10.

Key words and Phrases: electron scattering, ionization cross-sections, Hartree-Fock-Slater model. 
to a fundamental understanding of the nature. Despite the long-term study of the effects of electron scattering by atoms and ions, and the results obtained in the physics of atomic collisions, this area still requires theoretical and experimental studies; there is a significant lack of data on collision cross sections for their subsequent use in modeling and calculations. In particular, there are no systematized data on the cross sections for elastic scattering electrons by atomic ions and the differential ionization cross-sections of ions as well. All of these are required in Monte Carlo simulations for spectroscopic experiments to calculate correction factors and improve diagnostic methods. Also, detailed data on the cross sections of impact processes are needed when the particle transport modeling.

Experimental research methods in the field of interaction of electrons with atoms and ions have shown a significant improvement over the past decades due to the development of detectors and a decrease in delays in electronic components, which made it possible to expand the range of energies and angles of detection of interacting particles [5][6]. However, despite the progress made, the experimental data are still very limited, as well as the range of interaction parameters, and can mainly serve only for testing and calibration of theoretical models.

In parallel, the theoretical models and methods for calculating electron scattering cross sections have also been developed with an increase in the computational capability of modern computer systems. Over the past ten years, it has become possible to carry out individual calculations using rather complex second-order methods of perturbation theory, such as the close-coupling approximation [7] and R-matrix method [8] with various variations (including the R-matrix method with pseudo-state approximation and spline-representation of basic functions [9][10]). These methods involve solving a system of equations for selected bound states, and the convergence in solving such system of equations sharply decreases with an increase in the number of states. Thus, a reasonable limitation on the number of scattering channels arises and it is necessary to select the states to be considered, which requires an individual and careful approach when changing the ion charge, element, energy, etc. An incorrect or insufficient choice of such states leads to the loss of the most advantages from the use of complex methods, as well as to various artificial effects, for example, to pseudoresonances observed in the results of calculations [10][11]. Despite the difficulties, the abovementioned methods find their application in separate calculations of the resonance structure of cross sections and differential cross sections in the region of low energies of incident electrons (on the order of several electron-volts for atoms and first ions). Along with the quantum defect model, these methods have alterable success in accordance with the experimental results [12].

Various approximations and empirical formulas for calculating cross sections for collisional processes can be found in the open access [7][13]. However, such formulas have a limited scope of applicability, there are also significant differences in the results between them, and there are often sensible deviations from the experimental data. A known and available method for calculating cross sections is the Born approximation and its variations [14][15]. The conditions for its applicability arise when the energy of the scattered particle exceeds the potential energy of the external bound electrons by a factor of tens. In combination with the use of the screened potential of the ionic core, the Born approximation sometimes can give satisfactory agreement with experimental data even at low energies [16].

To describe the states of the plasma of multiply charged ions and the rates of atomic processes in a wide range of temperatures and densities, and to obtain reliable results, one 
should use sufficiently perfect quantum-statistical models of matter, for example, such as the Hartree-Fock-Slater (HFS) self-consistent model [17][18]. The HFS model uses the selfconsistent Hartree field and the local exchange approximation, which refines the Slater exchange potential. As a result of solving the Schrödinger equation with the obtained selfconsistent potential, the energy structure of an atom or ion can be found, and the corresponding wave functions calculated.

In this work, a technique is considered and proposed for calculating the cross sections for both elastic and inelastic scattering of electrons by atoms and ions. The described approach is based on the method of partial waves for the elastic and distorted wave approximation for the inelastic scattering, and the semiclassical approximation [7][19][20] using the self-consistent Hartree-Fock-Slater (HFS) potential [21], is characterized by a wide range of applicability and a moderate computational load. It allows one to obtain differential and integral cross-sections, as well as transport cross-sections with an accuracy, sufficient for use in calculating diffusion coefficients, thermal conductivity, etc., and modeling of the transport phenomena. Ehe ionization is the most complex process in calculational aspect, and the computation of the other inelastic processes can be analogically done basing on the same approach [22].

\section{ELASTIC ELECTRON SCATTERING ON ATOMS AND IONS}

In the expansion of the quantum theory of scattering into partial waves, the differential cross section for elastic scattering of a particle with a wavenumber $k$ on the central potential field can be expressed in terms of the scattering amplitude $A(\theta)$ in the solid angle $d \Omega$ and the phase shift $\delta_{l}[19][20]$

$$
\begin{gathered}
d \sigma=|A(\theta)|^{2} d \Omega \\
A(\theta)=\frac{1}{k} \sum_{l=0}^{\infty}(2 l+1) P_{l}(\cos \theta) \mathrm{e}^{i \delta_{l}} \sin \delta_{l} .
\end{gathered}
$$

After integration over the angle, the integral cross section of elastic scattering $\sigma$ can be obtained as

$$
\sigma=\int d \sigma=\frac{4 \pi}{k^{2}} \sum_{l=0}^{\infty}(2 l+1) \sin ^{2} \delta_{l}
$$

Herewith the orthogonality of Legendre polynomials $P_{l}(\cos \theta)$ for different orbital moments $l$ and $\int_{-1}^{1} P_{l}(\mu)^{2} d \mu=\frac{2}{2 l+1}$ are considered.

Numerous physical applications of collision theory (in particular, the particle transport in plasma), the transport cross section should be used instead of the integral cross-section [23]:

$$
\sigma_{t}=\int(1-\cos \theta) d \sigma=\frac{4 \pi}{k^{2}} \sum_{l=0}^{\infty}(2 l+1) \sin \delta_{l}\left[\sin \delta_{l}-\frac{2 l+2}{2 l+1} \sin \delta_{l+1} \cdot \cos \left(\delta_{l}-\delta_{l+1}\right)\right] .
$$

Phase shifts can be calculated by solving the Schrödinger equation. The exact solution of the scattering problem is possible only in certain types of potentials, in particular, for a 
Coulomb field, and in a more general case, the perturbation theory (Born approximation) or the WKB method (semiclassical approximation) have to be applied [19][24].

The Born approximation is applicable for a small phase shift relative to the phase of the wave function of free motion, i.e., at a low potential of the scattering field, or in the case of fast particles, so that

$$
|U|<<\frac{\hbar^{2}}{m a} k=\frac{\hbar p}{m a}=\frac{\hbar v}{a}=\frac{\hbar}{a} \sqrt{\frac{2 \varepsilon}{m}},
$$

where $a$ is the radius of action of the field $U(r), \mathrm{m}, \varepsilon, \mathrm{p}, \mathrm{v}$ are the reduced mass, energy, momentum and velocity of the incident particle, respectively.

In the semiclassical case, the wave functions are characterized by a large value of their phase

$$
\phi=\frac{\pi}{4}+\frac{1}{\hbar} \int_{r_{l}}^{r} p d r>>1 .
$$

The passage to the limit to the semiclassical approximation in the scattering theory corresponds to large phase shifts with respect to the phase of the wave function of free motion at $r \rightarrow \infty$

$$
\delta_{l}=\int_{r_{i}}^{\infty}\left[\frac{1}{\hbar} \sqrt{2 m(\varepsilon-U)-\frac{\hbar^{2}(l+1 / 2)^{2}}{r^{2}}}\right] d r-\int_{r^{\prime}}^{\infty}\left[\frac{1}{\hbar} \sqrt{2 m \varepsilon-\frac{\hbar^{2}(l+1 / 2)^{2}}{r^{2}}}\right] d r .
$$

Here $r_{l}$ and $r_{l}{ }^{\prime}$ are the turning points of the particle with the moment corresponding to the impact parameter $\rho=\frac{(l+1 / 2)}{k}$, in the presence and absence of a potential field, respectively. In the case of a multicharged ion, the field turns into a self-consistent field of the electrons $V(r)$ in the field of the uncompensated charge $Z_{i}$ of the ion at distances of the order of the size of the orbits of bound electrons, which transforms with increasing $r$ into a slowly decaying purely Coulomb field:

$$
U(r)=\left\{\begin{array}{ll}
V(r)-\frac{Z_{i} e^{2}}{r}, & r \leq r_{\max } \\
-\frac{Z_{i} e^{2}}{r}, & r>r_{\max }
\end{array} .\right.
$$

Due to the long-range action of the resulting ion potential, the main contribution to the elastic scattering cross section is made by the partial cross section at large moments $l$, where the semiclassical approximation is applicable. However, the direct application of formula (4) in (1) and (2) is associated with difficulties due to the divergence of the integrals.

For a purely Coulomb potential $U(r)=-\frac{Z_{i} e^{2}}{r}$ for all $r$, the direct solution of the Schrödinger equation leads to the Rutherford formula for the differential cross section for elastic electron scattering [25] 


$$
d \sigma=\left(\frac{Z_{i} e^{2}}{2 \varepsilon}\right)^{2} \frac{d \Omega}{4 \sin ^{4}(\theta / 2)} .
$$

Differential cross section (6) grows significantly with a decrease of the scattering angle, which is a result of the long-range field of the ion. For an ion potential like (5), the influence of the potential of the electrons should become negligible in comparison with the Coulomb potential of the ion at sufficiently small scattering angles and for the impact parameters of the incident electron more than $r_{\max }$. The differential cross section for such a potential should transform into Rutherford's formula (6) with a decrease in the scattering angle as well. Therefore, it is proposed to calculate the differential cross section for the ion potential (5) in two stages:

1. The differential cross section $d \sigma_{i}$ is calculated in the partial waves approach (1) in the semiclassical approximation framework using the formula for the phase shift (4) at $r \leq r_{\max }$ as a function of $r_{\text {max }}$;

2. The obtained cross section is joined with Rutherford's formula (6) at the scattering angle $\theta_{C} \ll<1$, corresponding to the impact parameter $\rho_{C}=r_{\max }=\frac{\left|Z_{i} e^{2}\right|}{2 \varepsilon} \frac{\cos \left(\theta_{C} / 2\right)}{\sin \left(\theta_{C} / 2\right)}$, which follows from the equation

$$
d \sigma=2 \pi \rho d \rho=\left(\frac{Z_{i} e^{2}}{2 \varepsilon}\right)^{2} \frac{2 \pi \sin (\theta) \mathrm{d} \theta}{\sin ^{4}(\theta / 2)}=8 \pi\left(\frac{Z_{i} e^{2}}{2 \varepsilon}\right)^{2} \frac{d(\sin (\theta / 2))}{\sin ^{3}(\theta / 2)} .
$$

From the "joining", the values $r_{\max }$ and $\theta_{C}$ are found in an implicit form. Thus, this approach should provide a correct approximation of the differential cross section for elastic scattering of electrons by multicharged ions.

The resulting differential cross section in the interval and its continuation according to Rutherford's formula (7) in the interval allow calculating the total transport cross section

$$
\sigma_{t}=\int(1-\cos \theta) d \sigma=16 \pi\left(\frac{Z_{i} e^{2}}{2 \varepsilon}\right)^{2} \int_{0}^{\theta_{c}} \frac{d(\sin (\theta / 2))}{\sin (\theta / 2)}+16 \pi \int_{\theta_{c}}^{\pi}\left(\frac{d \sigma_{i}}{d \Omega}\right) \sin ^{3}(\theta / 2) d(\sin (\theta / 2)) .
$$

The first integral in (8) diverges logarithmically at the lower integration limit, which is associated, as is known, with the long-range action of Coulomb forces, which leads to a high probability of scattering at small angles. However, in fact, in quasi-neutral plasma, the particle charge is screened by other charged particles at the Debye radius $r_{D}$. Therefore, electrons incident on an ion with an impact distance exceeding the Debye radius do not experience the action of its field. Taking this fact into account, we rewrite the first integral in (8), passing from the scattering angle to the impact parameter according to the relation $\rho=\frac{\left|Z_{i} e^{2}\right|}{2 \varepsilon} \frac{\cos (\theta / 2)}{\sin (\theta / 2)}$ : 


$$
16 \pi\left(\frac{Z_{i} e^{2}}{2 \varepsilon}\right)^{2} \int_{0}^{\theta_{c}} \frac{d(\sin (\theta / 2))}{\sin (\theta / 2)} \rightarrow 16 \pi \int_{\rho_{C}}^{r_{D}} \frac{\rho d \rho}{\left(\frac{\rho 2 \varepsilon}{Z_{i} e^{2}}\right)^{2}+1} \approx 16 \pi\left(\frac{Z_{i} e^{2}}{2 \varepsilon}\right)^{2} \ln \left(\frac{r_{D}}{\rho_{C}}\right) .
$$

The second integral in (8) is finite and does not diverge at the lower limit. Due to the smallness of $\theta_{C}<<1$, its limit can be extended to zero. As a result, for this addition to the transport section, we can apply the expression (3):

$$
\sigma_{i t}=\int(1-\cos \theta) d \sigma_{i}=\frac{4 \pi}{k^{2}} \sum_{l=0}^{\infty}(2 l+1) \sin \delta_{l}\left[\sin \delta_{l}-\frac{2 l+2}{2 l+1} \sin \left(\delta_{l+1}\right) \cdot \cos \left(\delta_{l}-\delta_{l-1}\right)\right],
$$

where the phase shifts are calculated, for example, in the WKB approximation according to formula (4) with a limit on the radius of action of the potential (5) $r \leq r_{\max }$.

Finally, the total transport section can be expressed as following

$$
\sigma_{t}=\int(1-\cos \theta) d \sigma=16 \pi\left(\frac{Z_{i} e^{2}}{2 \varepsilon}\right)^{2} \ln \left(\frac{r_{D}}{\rho_{C}}\right)+\sigma_{i t} .
$$

The expressions given above do not require numerical integration of differential cross sections, which significantly speeds up the calculation time, and can be directly used to calculate the cross sections. At modern workstations, the time for calculation a value of the cross section for a given particle energy ranges from a fraction of a second to several seconds, depending on the electron energy (the number of terms in the sum in (10) increases with increasing energy).

\section{INELASTIC ELECTRON SCATTERING ON ATOMS AND IONS - IONIZATION}

Inelastic impact processes of electrons with atoms and ions do not have an exact analytical solution for multielectron systems, being one of the problems in the theory of many bodies. To calculate the cross sections of these processes using quantum mechanical models, the perturbation theory is used as a rule. The interaction of an incident electron with electrons bound in an ion can be considered as a small perturbation. This interaction is small compared to the interaction of the incident or bound electrons with the potential field of the ion.

In perturbation theory, the cross sections of electron-ion collisional processes calculated through the matrix elements. In the computations, such matrix elements are calculated by various methods using the wave functions of bound and free electrons through the oscillator strengths and Slater integrals.

In the first order of perturbation theory, the distorted waves approximation and its variation are the most universal and most general method for calculating the cross sections of electron ion impact processes in plasma [7][24]. In the approximation the wave function of the incident electron is not a plane wave, but it is considered that the ion field affects the motion of a free electron. The wave function of an electron is a solution of the Schrödinger equation in the field of an ion, considering the interaction according to the Hartree-Fock-Slater model [17]. As a rule, the one-electron approximation in a central symmetric potential field is used. After averaging over the moments in the $L S$-coupling approach, the expression for the differential cross section of ionization by electron impact with the transition of an ion from 
state $\gamma_{0}$ to state $\gamma$ (with the transition of a bound electron $n l$ into a continuous spectrum with energy $\varepsilon^{*}$ and moment $\left.\lambda^{*}\right)$ is represented as [21][24]

$$
d \sigma_{\gamma_{0} \gamma}=\sum_{\kappa}\left[d \sigma_{\kappa}^{\prime}\left(n \ell, \varepsilon^{*} \lambda^{*}\right)+d \sigma_{\kappa}^{\prime \prime}\left(n \ell, \varepsilon^{*} \lambda^{*}\right)\right] .
$$

Here $d \sigma_{\kappa}^{\prime}\left(n \ell, \varepsilon^{*} \lambda^{*}\right)$ and $d \sigma_{\kappa}^{\prime \prime}\left(n \ell, \varepsilon^{*} \lambda^{*}\right)$ are differential parts of the cross-sections, including direct and interferential, and exchange components respectively:

$$
\begin{gathered}
d \sigma_{\kappa}^{\prime}\left(n \ell, \varepsilon^{*} \lambda^{*}\right)=\frac{4 \pi^{3} a_{0}^{2}}{(2 \ell+1) k^{2}} \sum_{\lambda, \lambda^{\prime}} P_{\kappa}^{d}\left(P_{\kappa}^{d}-\sum_{\kappa^{\prime}} P_{\kappa^{\prime} \kappa}^{e}\right) d \varepsilon^{*}, \\
d \sigma_{\kappa}^{\prime \prime}\left(n \ell, \varepsilon^{*} \lambda^{*}\right)=\frac{4 \pi^{3} a_{0}^{2}}{(2 \ell+1) k^{2}} \sum_{\lambda, \lambda^{\prime}}\left(\sum_{\kappa^{\prime}} P_{\kappa^{\prime} \kappa}^{e}\right)^{2} d \varepsilon^{*}
\end{gathered}
$$

where $k$ is the momentum of an incident electron; $\lambda$ and $\lambda^{\prime}$ are quantum momenta of incident electron before and after the interaction.

The direct and exchange radial integrals are expressed as follows through 3-jm and 6-j Wigner coefficients and Slater integrals:

$$
\begin{aligned}
& P_{\kappa}^{d}=\sqrt{\frac{(2 \ell+1)\left(2 \lambda^{*}+1\right)(2 \lambda+1)\left(2 \lambda^{\prime}+1\right)}{2 \kappa+1}}\left(\begin{array}{ccc}
\kappa & \ell & \lambda^{*} \\
0 & 0 & 0
\end{array}\right)\left(\begin{array}{ccc}
\kappa & \lambda & \lambda^{\prime} \\
0 & 0 & 0
\end{array}\right) \times R_{n \ell, \varepsilon^{*} \lambda^{*} ; \varepsilon \lambda, \varepsilon^{\prime} \lambda^{\prime}}^{(\kappa)} \\
& P_{\kappa^{\prime} \kappa}^{e}=(-1)^{\kappa+\kappa^{\prime}} \sqrt{(2 \kappa+1)(2 \ell+1)\left(2 \lambda^{*}+1\right)(2 \lambda+1)\left(2 \lambda^{\prime}+1\right)} \times \\
& \times\left(\begin{array}{ccc}
\kappa^{\prime} & \ell & \lambda^{\prime} \\
0 & 0 & 0
\end{array}\right)\left(\begin{array}{ccc}
\kappa^{\prime} & \lambda & \lambda^{*} \\
0 & 0 & 0
\end{array}\right)\left\{\begin{array}{ccc}
\kappa^{\prime} & \ell & \lambda^{\prime} \\
\kappa & \lambda & \lambda^{*}
\end{array}\right\} R_{\varepsilon \lambda, \varepsilon^{*} \lambda^{*} ; n \ell, \varepsilon^{\prime} \lambda^{\prime}}^{\left(\kappa^{\prime}\right)}
\end{aligned}
$$

$R_{\alpha, \beta ; \gamma, \delta}^{(\kappa)}$ are so-called Slater's integrals including the radial components of single-electron wave functions of bound and free electrons before and after impact

$$
R_{\alpha, \beta ; \gamma, \delta}^{(\kappa)}=\iint R_{\alpha}\left(r^{\prime}\right) R_{\beta}\left(r^{\prime}\right) \frac{\min \left(r^{\prime}, r^{\prime \prime}\right)^{\kappa}}{\max \left(r^{\prime}, r^{\prime \prime}\right)^{\kappa+1}} R_{\gamma}\left(r^{\prime \prime}\right) R_{\delta}\left(r^{\prime \prime}\right) d r^{\prime} d r^{\prime \prime} .
$$

Radial components of electron wave functions $R_{x}(r)$ are the solutions of the Schrödinger equation with self-consistent Hartree-Fock-Slater potential $V(r)$ :

$$
-\frac{1}{2} \ddot{R}+\left[-V(r)+\frac{l(l+1)}{2 r^{2}}\right] R=\varepsilon R .
$$

To calculate the effective cross section for ionization of an atom or an ion, the sum of the differential cross sections for each momentum should be integrated over the energies of the ionized electron 


$$
\sigma_{\gamma_{0} \gamma}=\sum_{\lambda^{*}} \int_{0}^{\varepsilon-\varepsilon^{i}} \frac{d \sigma_{\gamma_{0} \gamma}}{d \varepsilon^{*}} d \varepsilon^{*},
$$

where $\varepsilon^{i}$ is the ionization energy of corresponding bound electron.

To calculate the impact ionization cross sections, it is required to know the following characteristics of an atom: wave functions of electrons, energy levels, and level populations. The interaction between electrons in an ion is considered in the self-consistent field model. The self-consistent field model is that for an approximate description of the system, wave functions can be introduced for each particle. In this case, the interaction with other particles is approximately considered by introducing a field averaged over the motion of the remaining particles of the system using their one-particle wave functions. One-particle wave functions must be consistent in the sense that, on the one hand, they are a solution to the Schrödinger equation for one particle moving in the mean field created by other particles, and on the other, these same one-particle wave functions determine the mean field potential in which particles are moving. In general, the distorted wave approximation within the described procedure allows one to reach the sufficient for correct particle transport modelling cross-section values.

\section{RESULTS AND DISCUSSION}

The approaches proposed in previous paragraphs for calculation of elastic scattering and ionization processes by an electron impact were used to compute the corresponding cross sections for neutral and singly ionized nitrogen and oxygen atoms. The self-consistent Hartree-Fock-Slater potential was used as the atomic potential for calculating the phase shift in (2) and (11) and in the solution of Schrödinger equation for the radial wavefunction of electron and energies of bound electrons.

\subsection{Elastic scattering}

Data on the elastic scattering of electrons by neutral atoms of various elements are presented in the literature and data banks such as ENDF [26] and NIST [27] and can be served to compare and validate the computational results. In Figure 1 and Figure 2 the results of a comparison are presented of the calculated integral and transport cross-sections with the results from open access for scattering by nitrogen and oxygen atoms, respectively. It can be seen that with an increase in the energy of a scattered electron, the integral and transport cross sections diverge - this is explained by the fact that the differential scattering cross section sharply decreases with an increase in the particle energy and scattering angle. At low energies, a significant fraction is scattering at angles greater than $\pi / 2$, then, starting from several tens of electron-volts, this contribution is no longer so noticeable, and scattering occurs mainly in a narrow range of angles.

In contrast to scattering by neutral atoms, the data on scattering by positively charged ions available for use are very poor and, as a rule, are reduced to experimentally obtained differential cross sections for some elements and low energies of the scattered electron, as well as a limited range of scattering angles. The theoretical results, often obtained as a result of the use of close-coupling methods and R-matrix approximations, are presented mainly in comparison with the experimental data mentioned. In the literature, it was not possible to find 
systematized data on integral or transport cross sections for nitrogen and oxygen ions suitable for comparison with the results of calculations by the partial wave approximation proposed.

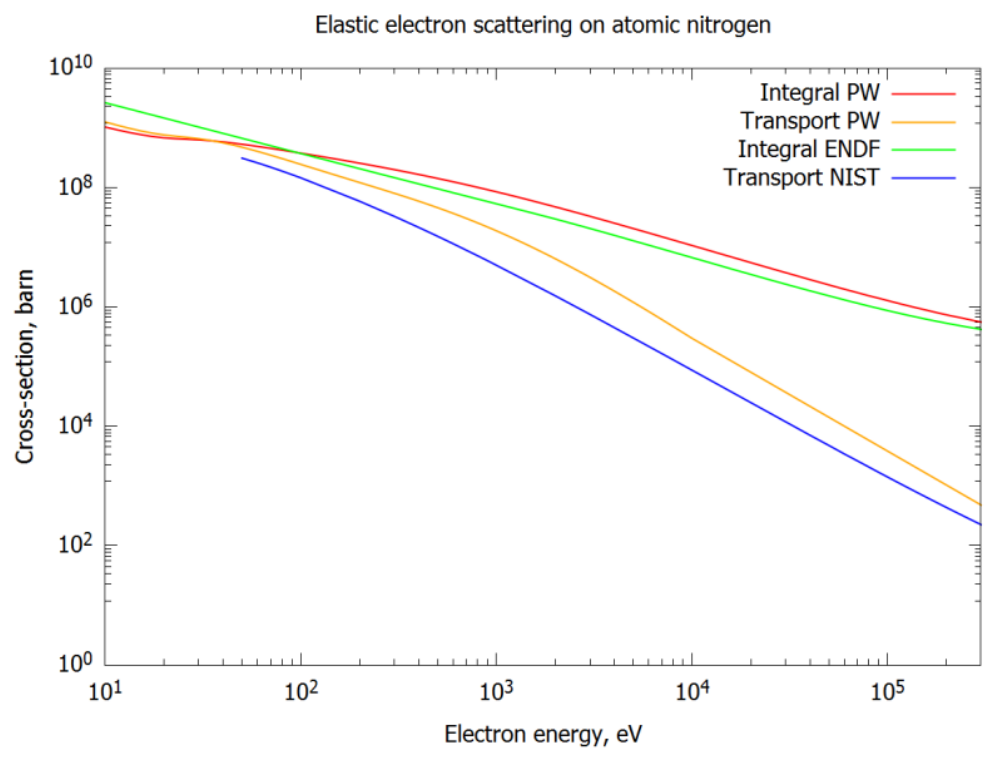

Figure 1: The calculated integral and transport cross sections for electron elastic scattering by a nitrogen atom as a function of the incident electron energy in comparison with the integral cross sections from the ENDF / B-VII database and the NIST data on the transport cross sections.

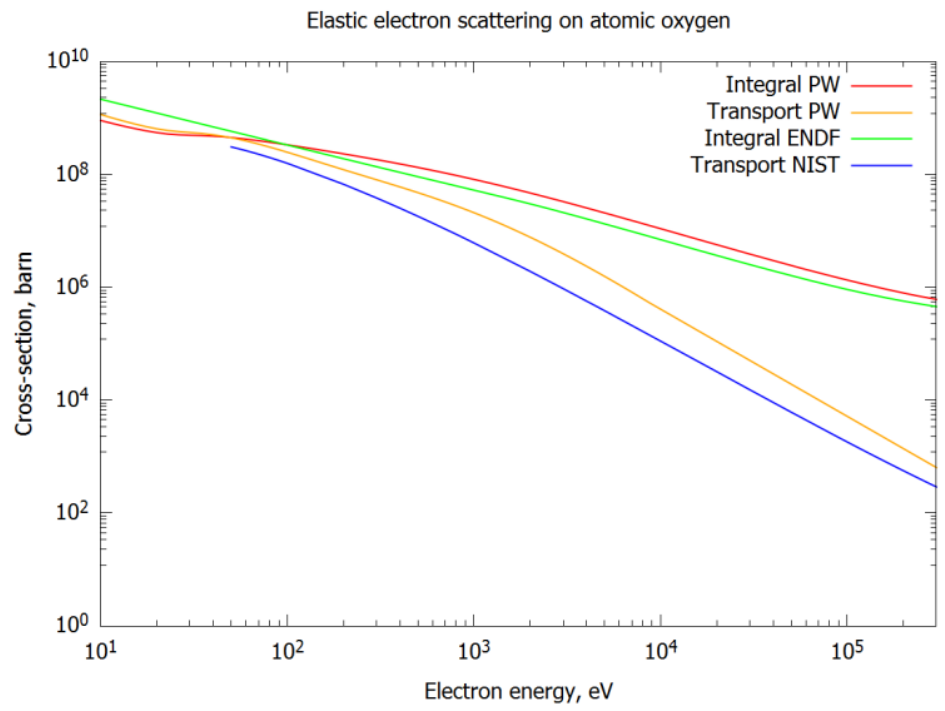

Figure 2: The calculated integral and transport cross sections for electron elastic scattering by an oxygen atom as a function of the incident electron energy in comparison with the integral cross sections from the ENDF / B-VII database and the NIST data on the transport cross sections 


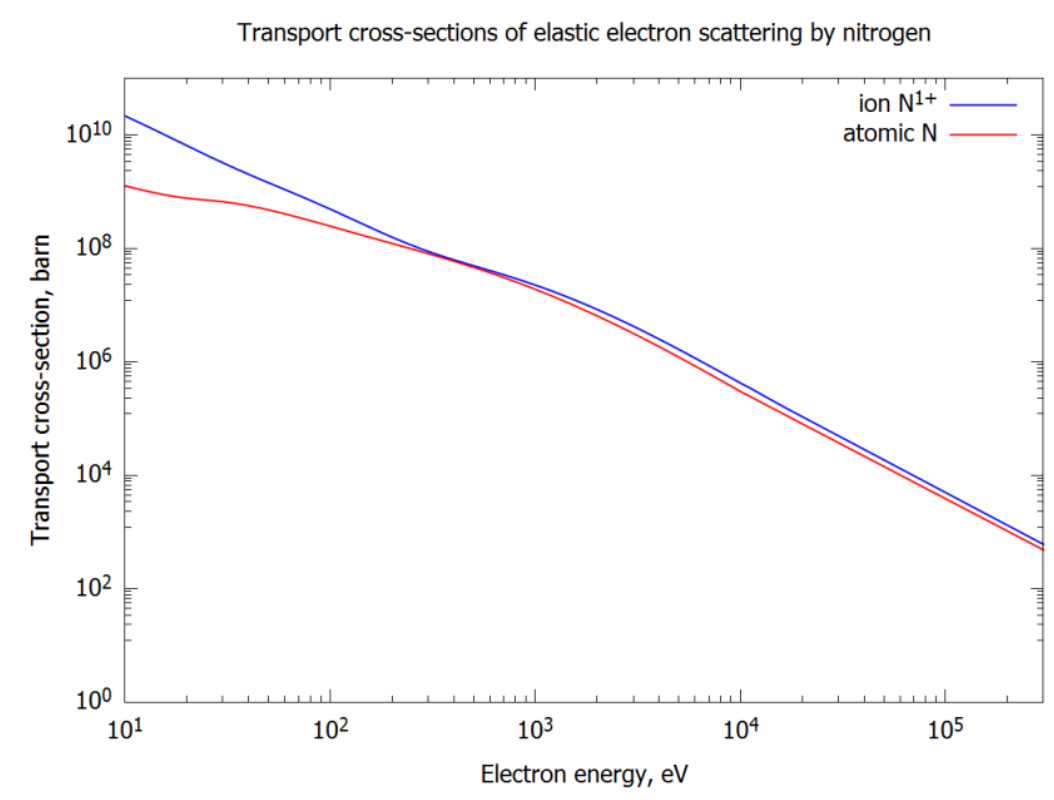

Figure 3: Transport cross section for elastic scattering by a neutral and singly ionized nitrogen

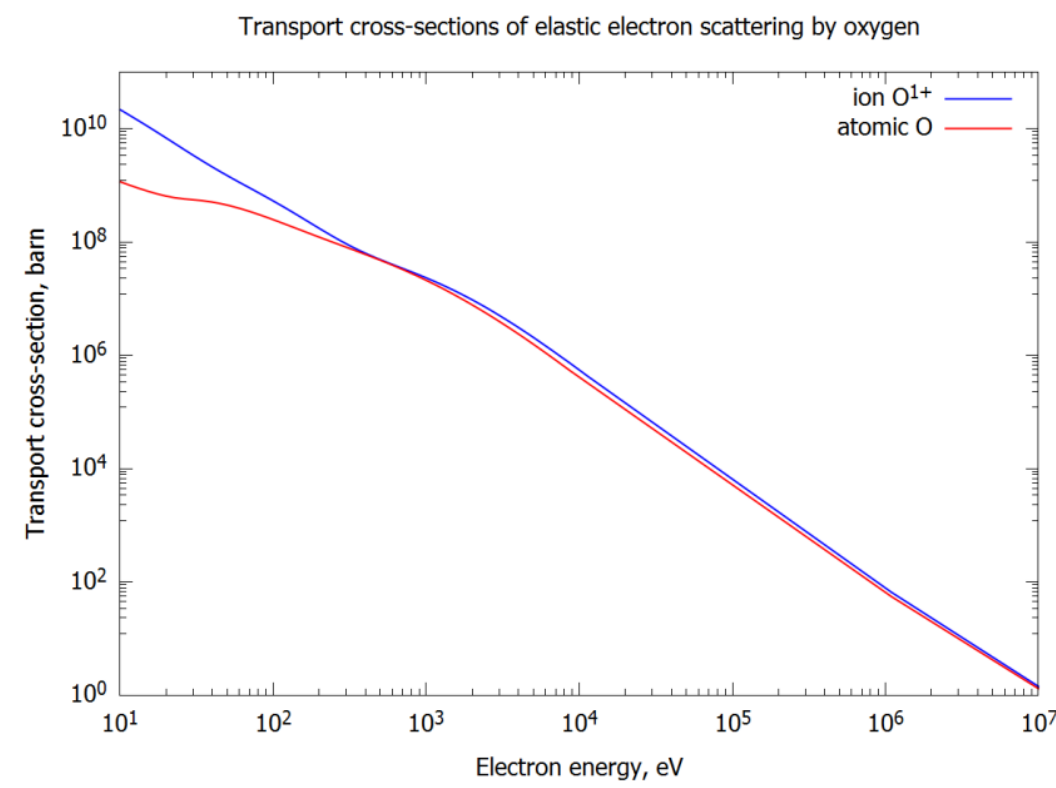

Figure 4: Transport cross section for elastic scattering by a neutral and singly ionized oxygen

The calculated transport cross sections for nitrogen and oxygen ions compared to the ones for atoms, respectively, shown in Figure 3 and Figure 4 demonstrate that the presence of a long-range potential significantly affects the range of electron scattering angles in a much wider energy range compared to the electron elastic scattering by a neutral atom. Scattering mainly occurs at a small angle, which leads to a multiple difference in the values of the transport cross sections even in the low-energy region. This fully corresponds to the theoretical concept described in the previous sections. 


\subsection{Ionization}

Within the scope of the transport modeling in a weakly ionized air, the differential cross sections and total cross sections of ionization for neutral atoms and single ions of nitrogen and oxygen were calculated in the approximation of distorted waves with wave functions computed in the Hartree-Fock-Slater potential. The obtained results are compared with the data on the differential and total cross sections for ionization of atoms by electron impact from the ENDF / B-VII.1 library and with the results of calculations using the binaryencounter approximation [28] and the semiclassical impulse approximation [29][30]. For the cross sections of ionization of $\mathrm{N}^{1+}$ and $\mathrm{O}^{1+}$ ions, the results of calculations are compared also with the recommended experimental data [31][32][33]. However, it should be remarked that the most of experimental data are of low accuracy, and the discrepancies in values between different experimental sets can reach 50\%. Also, many of the data presented are based on the approximation and extrapolation of experimental values to the high-energy region using classical approximations and empirical models.

As an example of calculation results of calculating the ionization cross-sections of electrons from inner $1 \mathrm{~s}$ and outer $2 \mathrm{p}$ subshells of oxygen atom are shown in Figure 5 - Figure 8. It can be seen that, considering the reference ENDF data, the distorted wave approximation, as a rule, describes the behavior of the cross sections better than other approximations. This is especially appreciable in the regions of electron energies that are less than several ionization energies in magnitude. In the absence of interference and exchange accounting, the cross sections obtained in the classical binary encounter approximation are being overestimated (Figure 7). Also, significant deviations from the reference data are observed for the cross sections calculated in the semiclassical momentum approximation (Figure 5, Figure 6 and Figure 8 ). The above remarks are also valid when calculating the cross sections for ionization of other elements.

In Figure 9 and Figure 10 one can see a comparison of the calculation results with the recommended and approximated experimental data for the total ionization cross sections of nitrogen and oxygen ions $\mathrm{N}^{1+}$ and $\mathrm{O}^{1+}$ respectively. The declared error of the experimental data and their approximation is $\pm 10 \%$. The cross sections resulted from the distorted wave approximation are in good agreement with the experimental data. It should be noted that a comparison of the ionization cross sections with experimental data requires careful analysis, since a significant contribution to the cross sections can be made by additional effects, such as the excitation of inner shells and subsequent autoionization. 


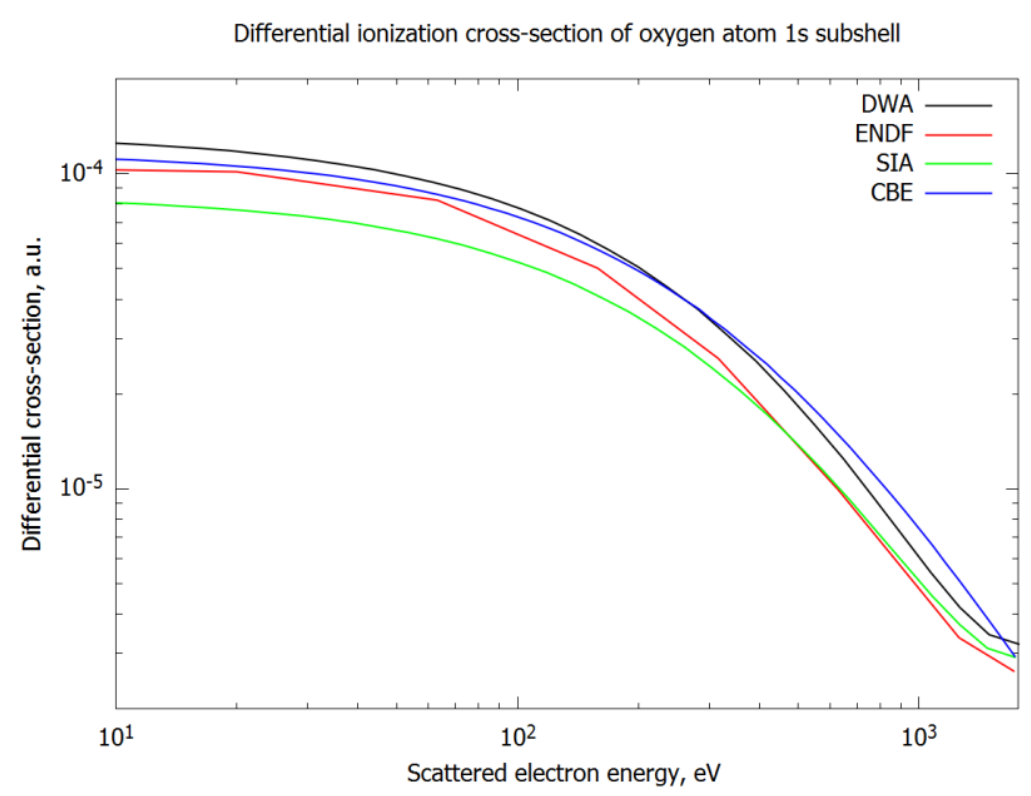

Figure 5: The differential ionization cross section of $1 \mathrm{~s}$ subshell in an oxygen atom for $3.9 \mathrm{keV}$ energy of impact electron, calculated by the distorted wave approximation, in comparison with the ENDF/B-

VII.1 data and the results of computations by the semiclassical impulse approximation and in the classical binary encounter approximation

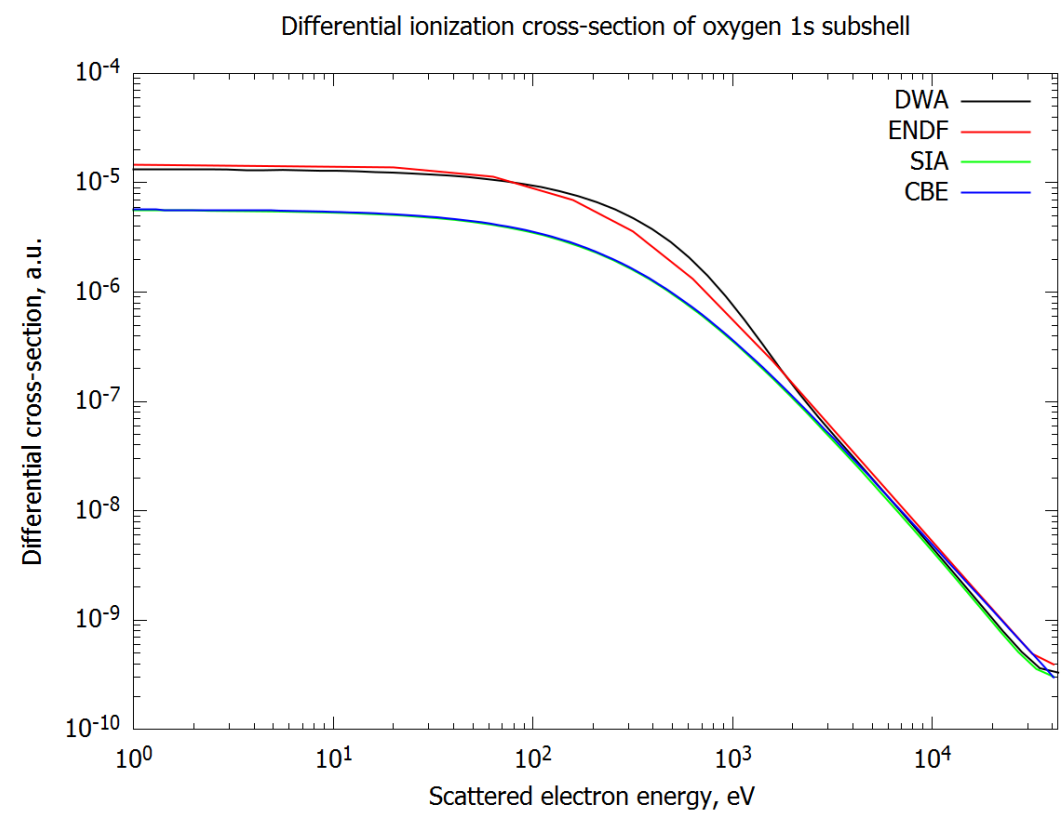

Figure 6: The differential ionization cross section of $1 \mathrm{~s}$ subshell in an oxygen atom for $81 \mathrm{keV}$ energy of impact electron, calculated by the distorted wave approximation, in comparison with the ENDF/BVII.1 data and the results of computations by the semiclassical impulse approximation and in the classical binary encounter approximation 


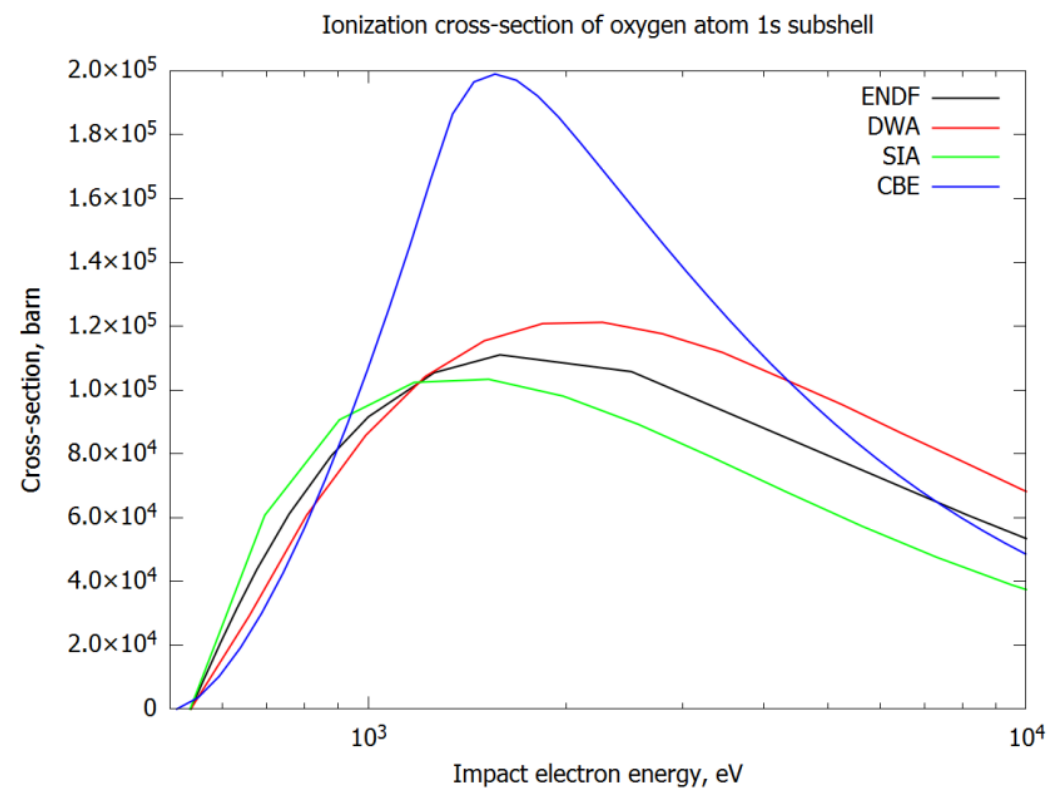

Figure 7: The ionization cross section of $1 s$ subshell in an oxygen atom, calculated by the distorted wave approximation, in comparison with the ENDF/B-VII.1 data and the results of computations by the semiclassical impulse approximation and in the classical binary encounter approximation

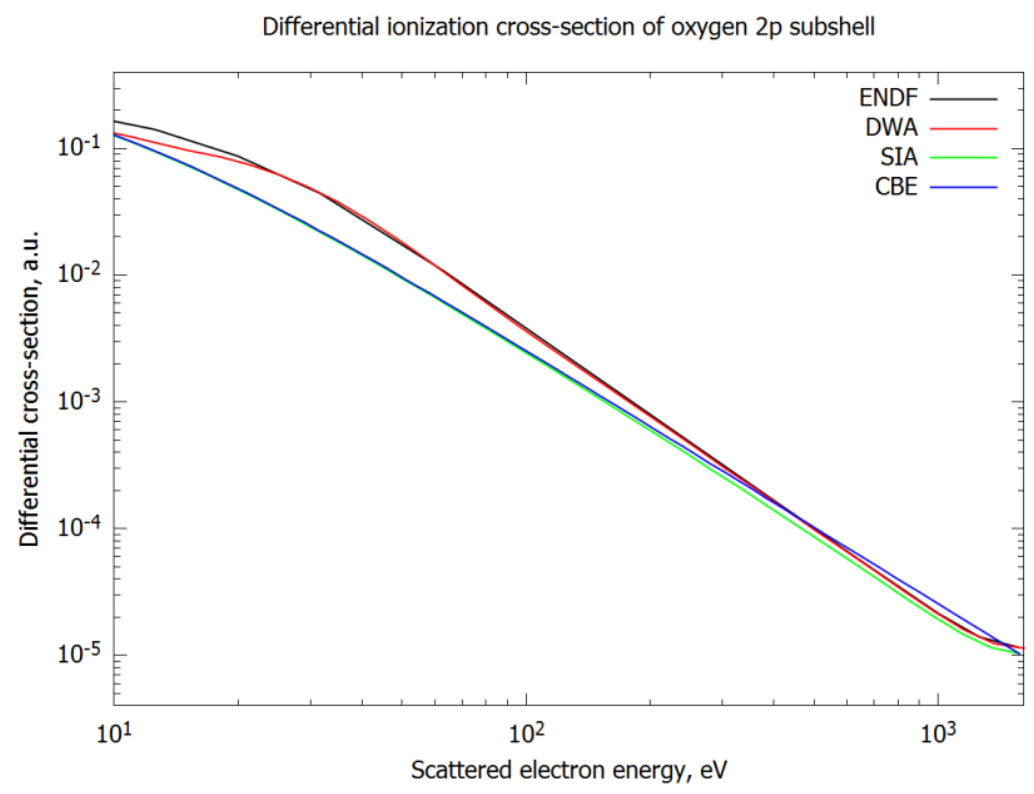

Figure 8: The differential ionization cross section of $2 p$ subshell in an oxygen atom for $3.1 \mathrm{keV}$ energy of impact electron, calculated by the distorted wave approximation, in comparison with the ENDF/B-

VII.1 data and the results of computations by the semiclassical impulse approximation and in the classical binary encounter approximation 


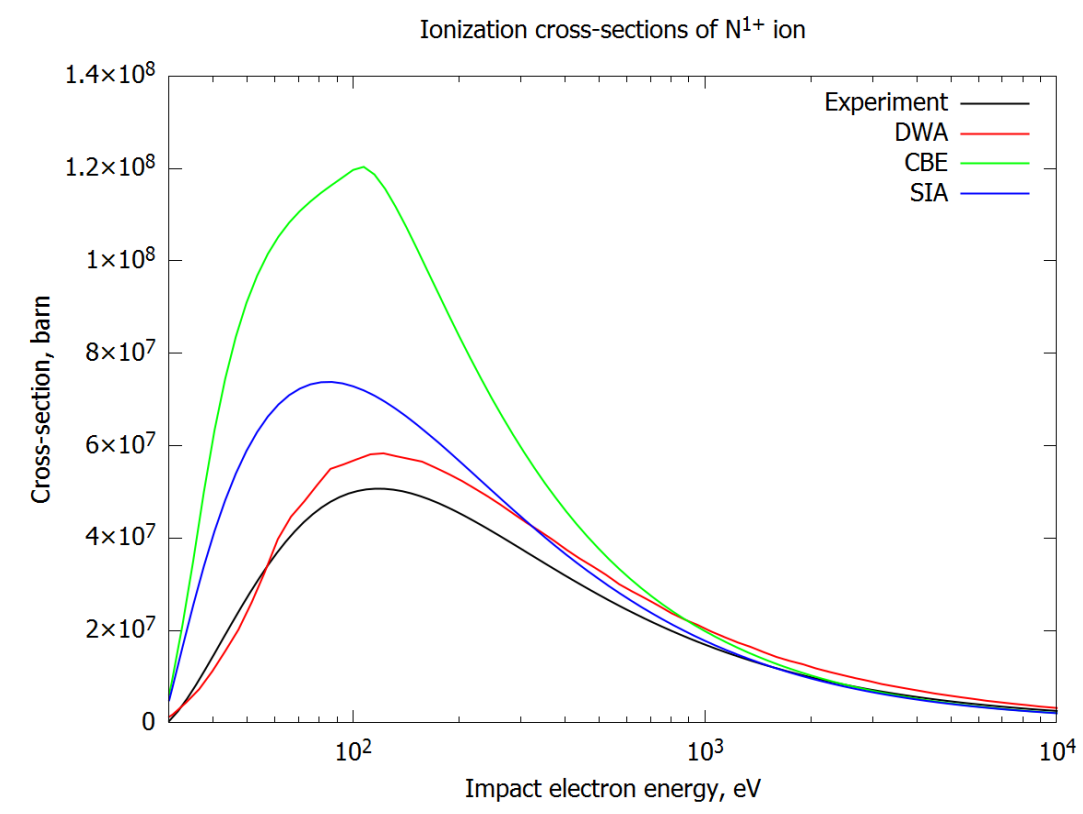

Figure. 9: The direct ionization cross section of a nitrogen ion $\mathrm{N}^{1+}$, calculated by the distorted wave approximation (red line), in comparison with the recommended experimental data [31][33] (black line) and the results of computations by the semiclassical impulse approximation (blue line) and in the classical binary encounter approximation (green line)

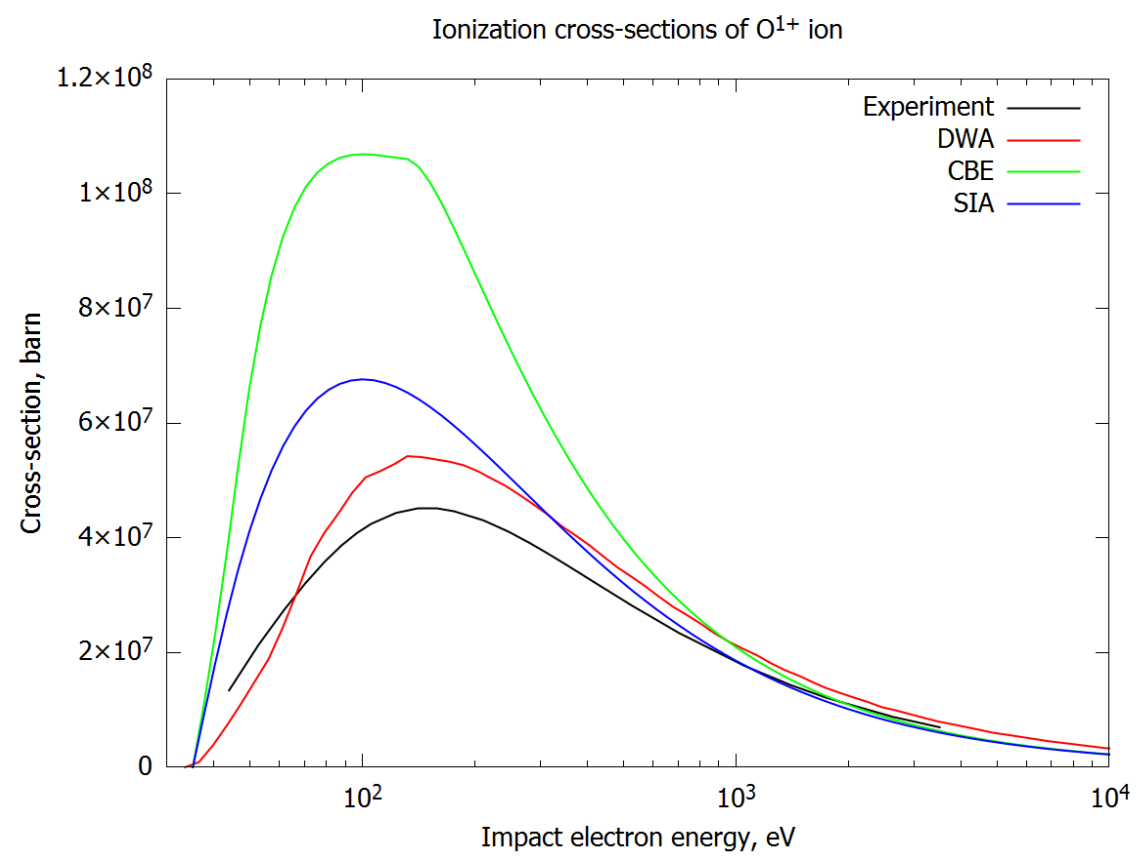

Figure 10: The direct ionization cross section of an oxygen ion $\mathrm{O}^{1+}$, calculated by the distorted wave approximation, in comparison with the recommended experimental data [32][33] and the results of computations by the semiclassical impulse approximation and in the classical binary encounter approximation 


\section{CONCLUSIONS}

A calculational approach for the cross-section computation of electron elastic scattering on neutral atoms and ions was discussed. The method is based on partial waves and semiclassical approximation with the use of self-consistent Hartree-Fock-Slater potential. The method is applicable in a wide range of electron energies, has a relatively low computational complexity, and allows the calculation of differential and total cross-section of elastic electron scattering, and the transport cross-sections needed as well. Decent accuracy of the approach permits to use the calculated data in diffusion, heat transfer etc computations and in the transport phenomenon modelling.

Calculated differential and total ionization cross-sections were compared to classical binaryencounter approximation, semiclassical impulse approximation and available experimental data. Results of calculations point to the computational approach proposed gives consistent results. It should be noted that in the collisional interaction of electrons with ions, the potential field of the latter can no longer be screened by the field of bound external electrons, and the effects of the potential on the incident particle are much stronger and cannot be omitted. Therefore, the use of the distorted wave approximation together with the Hartree-Fock-Slater quantum-statistical model is necessary for the correct calculation of the electron-ion interaction cross sections.

\section{REFERENCES}

[1] E. I. Popov, Spuskaemy`e apparaty, - M: Znanie, (1985).

[2] V.M. Donnelly, A. Kornblit, "Plasma etching: Yesterday, today and tomorrow", J. Vac. Sci. Technol., A 31(5), 050825 (2013).

[3] S.V. Zakharov, V.G. Novikov, P. Choi, "Z* Code for DPP and LPP Source Modeling", ed. V. Bakshi, SPIE: EUV Sources for Lithography, 223-275 (2006) doi: https://doi.org/10.1117/3.613774.ch8

[4] Th. von Woedtke, H.R. Metelmann, K.D. Weltmann, "Clinical plasma medicine: State and perspectives of in vivo application of cold atmospheric plasma", Contrib. Plasma Phys., 54(2), 104-117 (2014).

[5] F.H. Read, J.M. Channing, "Production and optical properties of an unscreened but localized magnetic field", Rev. Sci. Instrum., 67(6), 2372-2377 (1996).

[6] J. Ullrich, et al, "Recoil-ion and electron momentum spectroscopy: Reaction-microscopes", Rep. Prog. Phys., 66(9), 1463-1545 (2003).

[7] N.F. Mott, and H.S.W. Massey, The Theory of Atomic Collisions, 2nd ed., Oxford: The Clarendon Press, (1949).

[8] P.G. Burke and K.A. Berrington, Atomic and Molecular Processes: an R-Matrix Approach, Institute of Physics, Britstol, (1993).

[9] P.G. Burke, D.F. Gallaher, and S. Geltman, "Electron scattering by atomic hydrogen using a pseudo-state expansion I. Elastic scattering", J. Phys. B., 2, 1142 (1969).

[10] O. Zatsarinny and S.S. Tayal, "Low-energy electron collisions with atomic oxygen: R-matrix calculation with non-orthogonal orbitals", J. Phys. B., 34, 1299 (2001).

[11] M. Plummer, C.J. Noble, and M. Le Dourneuf, "Low-energy behaviour of e-O scattering calculations", J. Phys. B., 37, 2979 (2004).

[12] J.T. Shepherd and A.S. Dickinson, "Elastic scattering of electrons from positive ions", J. Phys. B: At. Mol. Opt. Phys., 32, 513 (1999).

[13] W. Lotz, "Electron-impact ionization cross-sections for atoms up to Z=108", Ztschr. Phys., 232, 101 (1970).

[14] H. A. Bethe and R. Jackiw, Intermediate Quantum Mechanics, Westview Press, (1986, 1997).

[15] H. Bethe, "Bremsformel für Elektronen relativistischer Geschwindigkeit", Zeitschrift für Physik, 76(5-6), 293-299 (1932). 
[16] J.B. Greenwood, I.D. Williams, B. Srigengan, W.R. Newell, J. Geddes, R.W. O'Neill, "Elastic scattering of electrons from ions", Nucl. Instr. and Meth. in Phys. Research Section B: Beam Interactions with Materials and Atoms, 98 (1-4), 125-128 (1995).

[17] Arnold F. Nikiforov, Vladimir G. Novikov, V. B. Uvarov, Quantum-Statistical Models of Hot Dense Matter and Methods for Computation Opacity and Equation of State, Basel: Birkhäuser Verlag, (2005).

[18] B.F. Rozsnyai, "Relativistic Hartree-Fock-Slater calculations for arbitrary temperature and matter density", Phys. Rev. A., 5(3), 1137 (1972).

[19] A.S. Davydov, Quantum Mechanics: International Series in Natural Philosophy. Elsevier, (1965).

[20] G.F. Drukarev, Collisions of Electrons with Atoms and Molecules. Springer US, (1987).

[21] V.G. Novikov, A.D. Solomyannaya, V.S. Zakharov, "Kvantovo-statisticheskie metody rascheta opticheskih i termodinamicheskih svojstv plotnoj plazmy”, Enciklopediya nizkotemperaturnoj plazmy VII, 2 (B), Matematicheskoe modelirovanie v nizkotemperaturnoj plazme, Ed. Fortov V.E., Moskva, YAnus-K, 378-435 (2008).

[22] V.S. Zakharov and V.G. Novikov, "Modeling of Ionization Composition in Argon Plasma with Fast Electrons", Math. Models Comput. Simul., 1, 533-542 (2009).

[23] Reviews of Plasma Physics. Editor: M. A. Leontovich, Vol. 1. Springer US (1980).

[24] I.I. Sobelman, L.A. Vainshtein and E.A. Yukov, "Excitation of Atoms and Broadening of Spectral Lines, Berlin: Springer-Verlag, 1981.E. Rutherford. The Scattering of $\alpha$ and $\beta$ Particles by Matter and the Structure of the Atom", Philos. Mag., 6, 21 (1909).

[25] E. Rutherford, "The Scattering of $\alpha$ and $\beta$ Particles by Matter and the Structure of the Atom", Philos. Mag., 6, 21 (1909).

[26] Evaluated Nuclear Data File (ENDF) Retrieval \& Plotting. https://www.nndc.bnl.gov/sigma (Accessed July 28, 2021).

[27] A. Jablonski, F. Salvat, C. J. Powell, and A. Y. Lee, NIST Electron Elastic-Scattering CrossSection Database Version 4.0, NIST Standard Reference Database Number 64, National Institute of Standards and Technology, Gaithersburg MD, 20899 (2016).

[28] L. Vriens. "Binary-encounter electron-atom collision theory", Phys. Rev., 141(1), 88 (1966).

[29] R. C. Stabler. "Classical impulse approximation for inelastic electron-atom collisions", Phys. Rev., 133, A1268-A1273 (1964).

[30] M. Gryziński. "Two-Particle Collisions. I. General Relations for Collisions in the Laboratory System", Phys. Rev., 138, A305, A332, A336 (1965).

[31] M.F.A. Harrison, K.T. Dolder and P.C. Thonemann, "A Measurement of the Cross Section for the Ionization of $\mathrm{N}^{+}$to $\mathrm{N}^{2+}$ by Electron Impact", Proc. Phys. Soc., 82, 368 (1963).

[32] K.L. Aitken and M.F.A. Harrison, "Measurement of the cross sections for electron impact ionization of multi-electron ions; I. $\mathrm{O}^{+}$to $\mathrm{O}^{2+}$ and $\mathrm{O}^{2+}$ to $\mathrm{O}^{3+»,}$ J. Phys. B, 4, 1176 (1971).

[33] K.L. Bell et al, «Recommended data on the electron impact ionization of light atoms and ions", J. Phys. Chem. Ref. Data, 12(4), 891-916 (1983).

Received Juny 20, 2021 